\title{
Operational forecast of hydrophysical fields in the Georgian Black Sea coastal zone within the ECOOP
}

\author{
A. A. Kordzadze and D. I. Demetrashvili \\ M. Nodia Institute of Geophysics, Iv. Javakhishvili Tbilisi State University, 1, M. Alexidze Str., 0173 Tbilisi, Georgia
}

Received: 31 December 2010 - Published in Ocean Sci. Discuss.: 21 February 2011

Revised: 26 August 2011 - Accepted: 1 November 2011 - Published: 25 November 2011

\begin{abstract}
One of the parts of the Black Sea Nowcasting/Forecasting System is the regional forecasting system for the easternmost part of the Black Sea (including the Georgian water area), which has been developed within the context of the EU International projects ARENA and ECOOP. A core of the regional system is a high-resolution baroclinic regional model of the Black Sea dynamics developed at M. Nodia Institute of Geophysics (RM-IG). This model is nested in the basin-scale model of Marine Hydrophysical Institute (MHI, Sevastopol/Ukraine). The regional area is limited to the Caucasian and Turkish coastal lines and the western liquid boundary coinciding with the meridian $39.36^{\circ} \mathrm{E}$. Since June 2010 we have regularly been computing 3 days' forecasts of current, temperature and salinity for the easternmost part of the Black Sea with $1 \mathrm{~km}$ spacing. In this study the results of two forecasts are presented. The first forecast corresponds to summer season and covers the prognostic interval from 00:00 h, 6 August to 00:00 h, 9 August 2010. The second one corresponds to autumn season and covers the prognostic interval from 00:00 h, 26 October to 00:00 h, 29 October 2010. Data needed for the forecasts - the initial and prognostic hydrophysical fields on the open boundary, also 2-D prognostic meteorological fields at the sea surface - wind stress, heat fluxes, evaporation and precipitation rates for our regional area are being placed on the MHI server every day and we are available to use these data operatively. Prognostic hydrophysical fields are results of forecast by the basin-scale model of MHI and 2-D meteorological boundary fields represent the results of forecast by regional atmospheric model ALADIN. All these fields are given on the grid of basin-scale model with $5 \mathrm{~km}$ spacing and with one-hour time step frequency for the integration period. The analysis of predicted
\end{abstract}

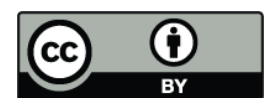

Correspondence to: D. I. Demetrashvili (demetr_48@yahoo.com) fields shows that to use the model with high resolution is very important factor for identification of nearshore eddies of small sizes. It should be noted the very different character of regional circulation in summer and autumn seasons in the easternmost part of the Black Sea.

\section{Introduction}

Large scientific and technical achievement of the Black Sea operational oceanography for the last decade is the development of the Black Sea Nowcasting/Forecasting System which allows to carry out continuous control over a current state of the Black Sea and its change for some days forward (Besiktepe, 2003; Korotaev and Eremeev, 2006). Creation of such system was promoted by the leading oceanographic Centers of the Black Sea riparian countries within the EU International scientific and technical projects ARENA and ECOOP. Coordination of this work was carried out by Marine Hydrophysical Institute (MHI, Sevastopol, Ukraine) of the National Academy of Sciences of Ukraine. Functioning of the system in operative mode is especially important for coastal and shelf areas which undergoes the greatest anthropogenous loading.

The main structure of the Nowcasting/Forecasting System is described by Korotaev et al. (2006) and Kubryakov et al. (2006). One of the parts of the System is the regional forecasting system for the easternmost part of the Black Sea (including the Georgian water area), the core of which is a high-resolution baroclinic regional model (RMIG) of the Black Sea dynamics developed at M. Nodia Institute of Geophysics by Kordzadze and Demetrashvili (2008, 2010). This model is nested in the basin-scale model (BSM) of the Black Sea dynamics of MHl (Demyshev and Korotaev, 1992). Note that the pilot near-real time operation of

Published by Copernicus Publications on behalf of the European Geosciences Union. 
the Black Sea Nowcasting/Forecasting System (including regional forecasting system for the Georgian water area) was successfully carried out for the first time in July 2005 during five days (Kubryakov et al., 2006; Kordzadze and Demetrashvili, 2008).

Since June 2010 at M. Nodia Institute of Geophysics 3 days' forecasts of 3-D fields of current, temperature and salinity with high resolution have been regularly carried out for the easternmost part of the Black Sea. All input data needed for initial and boundary conditions are available from the MHI server.

In this study we consider the regional forecasting system for the easternmost part of the Black Sea. To demonstrate the functioning of the system, two forecasts of hydrophysical fields for summer and autumn seasons are discussed.

\section{Regional forecasting system}

The main component of the regional forecasting system (RM-IG) is developed by adaptation of the BSM of the Black Sea dynamics of M. Nodia Institute of Geophysics (Kordzadze and Demetrashvili 2004; Kordzadze et al., 2008; Demetrashvili et al., 2008) to the easternmost part of the basin and one-way nesting in the BSM of MHI (without feedback from RM-IG to BSM). It is necessary to note that in turn the BSM of Institute of Geophysics is an improved version of the prognostic model of the Black Sea dynamics (Kordzadze and Skiba, 1973; Marchuk et al., 1975, 1979; Marchuk and Kordzadze, 1986; Kordzadze, 1989) originally developed in the early 1970s at the Computing Center of Siberian Branch of the Academy of Sciences USSR (Novosibirsk, Akademgorodok).

\subsection{Model description}

The RM-IG is based on a primitive system of ocean hydrothermodynamics equations in hydrostatic approximation, which is written in $z$-coordinates for deviations of thermodynamic values from their standard vertical distributions (Kordzadze and Demetrashvili, 2008, 2010).

The model equation system is given in Appendix A. The model takes into account: nonstationary atmospheric wind and thermohaline forcing, quasi-realistic bottom relief, the absorption of solar radiation by the sea upper layer, spacetemporal variability of horizontal and vertical turbulent exchange (Appendix B).

Atmospheric forcing is taken into account by boundary conditions on the sea surface which is considered as a rigid surface, where Neumann conditions are used by given wind stress components, heat fluxes, precipitation and evaporation from the sea surface. On the sea bottom the velocity components, heat and salt fluxes are equal to zero. On the lateral surfaces, two kinds of boundary conditions are considered: (1) on the rigid boundaries, sharing the sea from the land, components of current velocity, gradients of temperature and salinity normal to the boundary surface are equal to zero; (2) on the liquid boundary prognostic values of velocity components, temperature and salinity computed on the base of BSM of MHI are used.

\subsection{Method of solution}

The existence and uniqueness theorems of 3-D nonstationary problem of the sea dynamics are proved by Sukhonosov (1981) and Kordzadze (1982). To solve the problem we used the two-cycle method of splitting the model equation system with respect to both physical processes and coordinate planes and lines, which was proposed to solve problems of ocean and atmosphere dynamics by Marchuk $(1967,1974)$.

Let us describe the numerical algorithm briefly. After splitting the model equation system (see Appendix A) with respect to physical processes the following main stages are allocated: (1) the transfer of the physical fields taking into account eddy viscosity and diffusion; (2) the adaptation of the physical fields with division of the solution into barothropic and baroclinic components. For approximation on time of all split problems the Krank-Nickolson scheme is used.

At the transfer stage, after using two-cycle splitting method with respect to coordinates and corresponding transformation, we receive one-dimensional finite-difference equations on coordinates $x, y$, and $z$ for grid functions of velocity components $u$ and $v$, temperature deviations $T$ and salinity deviations $S$. The received equations are solved by the factorization method.

At the adaptation stage the barothropic and baroclinic components are allocated. The barothropic task is reduced to solution of 2-D finite-difference equations for integral stream function.

The differential operator of the baroclinic problem is preliminary splitting with the purpose of allocating the term describing the Coriolis force action as an independent stage. After using finite-difference approximation the remaining part of the differential operator of the baroclinic problem splits into vertical coordinate planes $z x$ and $z y$. As a result, a set of 2-D problems for baroclinic components on vertical planes are received, which then also are reduced to the equations for analogs of stream functions.

Thus, the adaptation problem as a whole is reduced to solution of sequence of the same type 2-D problems for the stream function and analogs of stream functions, which are efficiently realized within the framework of uniform iterative algorithm (Kordzadze, 1989). The exception is a task considering the Coriolis force action, which is explicitly realized by solution of algebraic equations. At the transfer stage all required grid functions are defined in grid points with integer indexes, and at the stage of adaptation the functions are defined on the shifted grids. Transition from one grid to 
another is carried out by the linear interpolation at each time step.

The finite-difference schemes received at each elementary stage of splitting are absolutely steady, energetically balanced and provide the second order accuracy on time and space coordinates (in case of uniform grid). The use of the splitting method substantially simplifies the implementation of complex physical model and enables us to reduce solution of 3-D nonstationary problem to solution of more simple 2-D and 1-D problems.

\subsection{Methodology of nested grid modeling and some input parameters}

The high-resolution RM-IG covers the regional area bounded with the Caucasus and Turkish shorelines and the western liquid (open) boundary coincident with $39.36^{\circ} \mathrm{E}$ with a grid having $193 \times 347$ points on horizons (grid step $1 \mathrm{~km}$ ). On a vertical the non-uniform grid with 30 calculated levels on depths: $2,4,6,8,12,16,26,36,56,86,136,206,306, \ldots$, $2006 \mathrm{~m}$ are considered. The time step is equal to $0.5 \mathrm{~h}$.

The RM-IG is nested in the BSM of MHI with grid step $5 \mathrm{~km}$. In addition, there is applying one-way nesting, which provides forcing of basin-scale processes on the regional processes via the open boundary. Data needed for the regional forecasts - the 3-D initial and 2-D prognostic fields of velocity components, temperature and salinity on the open boundary, also 2-D prognostic meteorological fields at the sea surface - wind stress, heat fluxes, evaporation and precipitation rates for our regional area are providing from MHI via ftp site and we are available to use these data operatively. Prognostic hydrophysical fields on the open boundary are results of forecast from BSM of MHI and 2-D meteorological boundary fields represent results of forecast from the regional atmospheric model ALADIN. All these fields are given on the grid of BSM with one-hour time step frequency within the 4-days period. With the purpose of using the received data as initial and boundary conditions (on upper and open boundaries), during model implementation these fields are transferred to grid of RM-IG with $1 \mathrm{~km}$ spacing by interpolation. Thus, on each time step on the western liquid boundary we have forecasted values of flow velocity components, temperature and salinity which are used as open boundary conditions for the RM-IG. It should be noted that via ftp site we obtain also 3-D hydrophysical fields forecasted from BSM of $\mathrm{MHI}$ and corresponding to our regional domain. This fact enables us to compare results of forecasts, obtained from both RM-IG and BSM of MHI.

Concerning the input data it is necessary to note the following. The input data, which are given on a course grid, provide to run for 4-days, but we consider that the RM-IG gives forecast only for three days as during the first day the coastal model runs in the prognostic mode only to have better adjustment of the fine resolution to the coarse initial conditions provided by the BSM of MHI.
The software of the problem is developed on the basis of the algorithmic language "Compaq Visual Fortran 6.1", and realization of the forecasting system is carried out by means of PC Pentium-4 with $3.00 \mathrm{GHz}$. The system allows to calculate 3-D fields of current, temperature, salinity and density in the Black Sea coastal zone with $1 \mathrm{~km}$ resolution. At 24, 48, and $72 \mathrm{~h}$ after starting the forecast visualization of the flow, temperature and salinity fields on horizons $0,10,20,50,100$, $200,500,1000 \mathrm{~m}$ is carried out using the System software "Surfer- 8 ". Transition from calculated levels to these ones is carried out by the linear interpolation.

\section{Simulation and forecast of regional circulation processes}

To demonstrate the operation of the regional forecasting system, there are considered two examples of the forecast corresponding to summer and autumn seasons, when circulating features of waters of the Georgian Black Sea coastal zone area extremely differed from each other. Before to analyze forecast results, simulated fields of SST are compared with satellite SST.

\subsection{Validation of simulated SST}

With the purpose of validating the regional system comparison of predicted fields with real data have been done during the pilot experiment within the project ARENA in 2005, which showed an ability of the RM-IG to predict hydrophysical fields with sufficient accuracy (Kubryakov et al., 2006). In this study we have carried out comparison of simulated SST corresponding to 2010-2011 with satellite images SST derived from NOAA. The remote-sensing data were available from the Black Sea data archive of the National Space Agency of Ukraine (http://dvs.net.ua/mp). Mostly the days were selected when the sky was cloud-free over the easternmost Black Sea. The analysis of results of comparison showed a good qualitative correspondence between forecasted and observed temperature fields. Quantitative difference in many cases does not exceed $0.7-0.8^{\circ} \mathrm{C}$.

Some examples of validation of forecasted SST are shown in Fig. 1. Here periods August and October 2010 are chosen, which are considered in the next subsection to analyze forecast results. Fig. 1a and c correspond to forecast start at 5 August 2010, 00:00 h (here and after local time is used), and Fig. 1e - to forecast start at 25 October 2010, 00:00 h. It is necessary to note that because of using of different color scales (for example, satellite images are given in continuous spectrum while simulated SST - in discrete spectrum) close similarity between the forecasted and satellite SST at once is not obvious visually, but their careful analysis shows, that simulated and observed temperature fields are in a good agreement to each other. For example, as a result of comparison of Fig. 1a and b it is easy to notice that both 


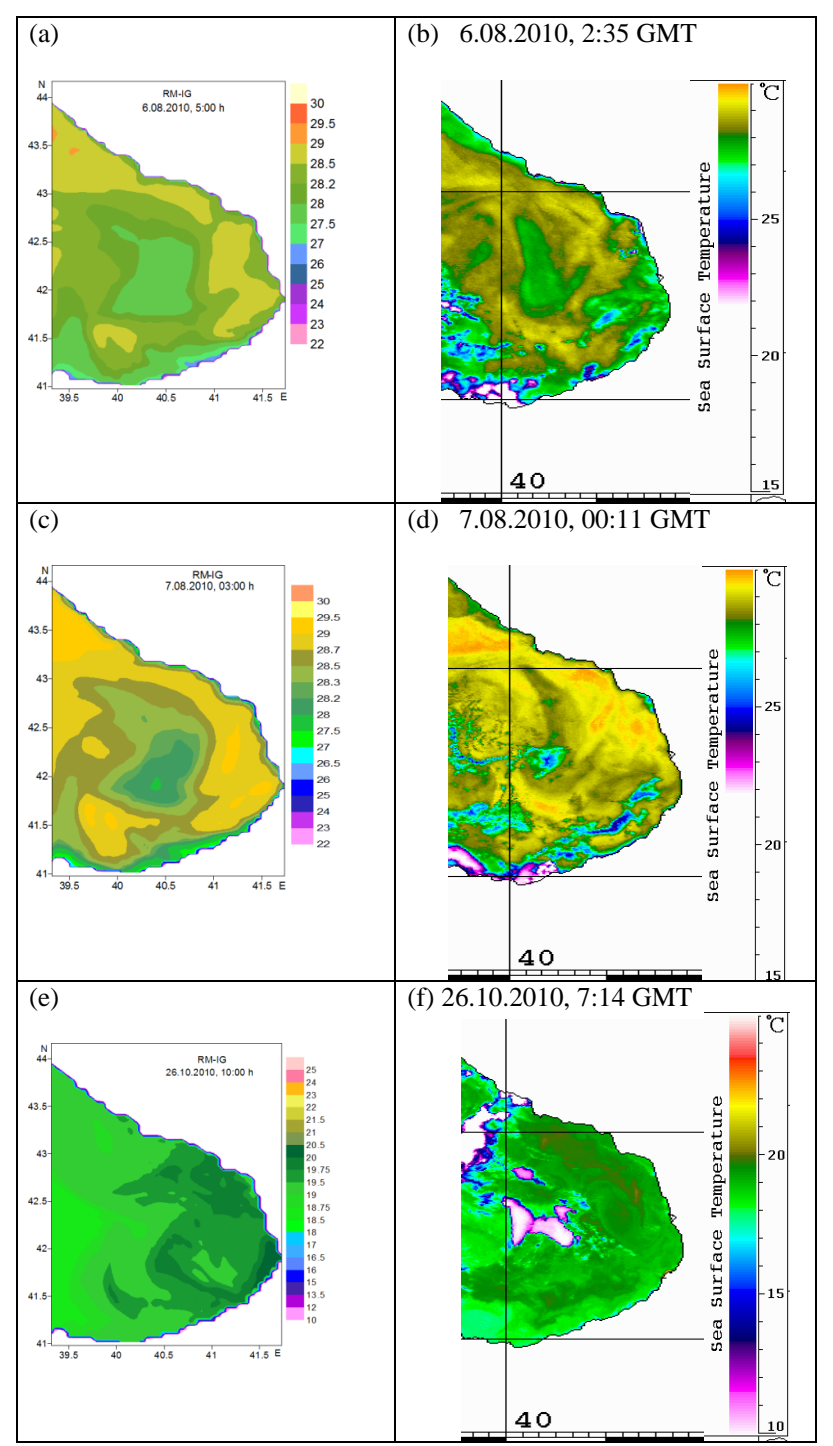

Fig. 1. Forecasted (on the left side) and satellite SST derived from NOAA (on the right side).

the simulated and measured SST patterns equally reflect the main features of the temperature field, for example, alongshore water transport with relatively high temperature and a zone with relatively lower temperature approximately in the central part of the considered area. The same can be noted at comparison between Fig. 1c and d.

It should be noted that in October for the forecasting period presented here cloudiness dominated over the Black Sea including the easternmost part. Only on 26 October 2010 the sky was relatively cloud-free over the easternmost part of the sea basin but in this case some clouds in the central and north-western parts of the basin were observed too (Fig. 1f). According to simulated and observed data (Fig. 1e and f) a common tendency of water cooling is observed in process of increase of distance from the sea coast. Concerning quanti-

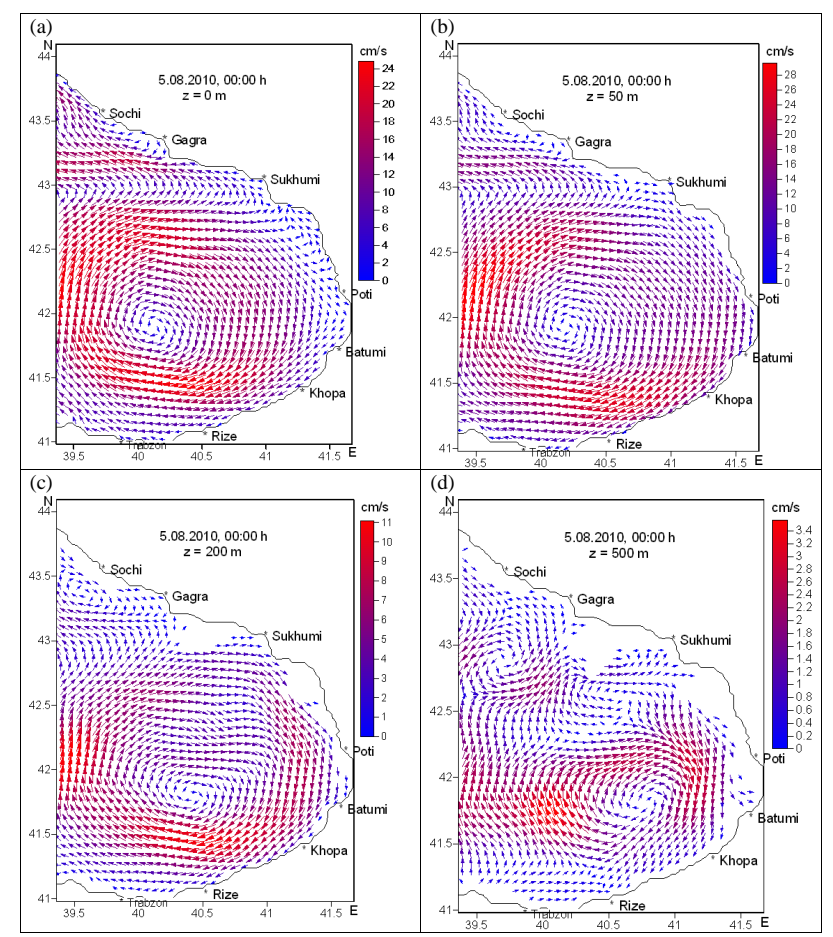

Fig. 2. Current field at 5 August 2010, 00:00 h on depths of $0 \mathrm{~m}$ (a), $50 \mathrm{~m} \mathrm{(b),} 200 \mathrm{~m} \mathrm{(c),} \mathrm{and} 500 \mathrm{~m}$ (d).

tative comparison, in the most part of the considered domain differences between forecasted and observed values of SST do not exceed $0.5^{\circ} \mathrm{C}$, but there are some points where are significant differences.

\subsection{Forecast for summer season}

The forecasting time period lasted from 00:00 h, 6 August to 00:00 h, 9 August 2010, but according to discussed above, integration of model equations began one day earlier, i.e. at $00: 00 \mathrm{~h}, 5$ August. At this moment the current field in the regional area on the different depths is shown in Fig. 2. From this Figure it is clear that the main element of the regional circulation is the anticyclonic eddy (called the Batumi eddy) with diameter about of 150-200 km, which covers the significant part of the considered area. This vortex practically does not change in the upper $200 \mathrm{~m}$ layer, only current speed decreases from maximal value equal to $24 \mathrm{~cm} \mathrm{~s}^{-1}$ till $11 \mathrm{~cm} \mathrm{~s}^{-1}$, below $200 \mathrm{~m}$ the vortex gradually decreases in sizes (Fig. 2d). At deeper levels the Batumi eddy gradually disappeared.

The temperature and salinity fields on the Black Sea surface and horizon $z=100 \mathrm{~m}$ at the beginning of integration of model equation system are presented in Figs. 3 and 4. Horizontal distribution of the surface temperature is typical for the summer period, warmer waters are in the nearshore zone to Caucasus sealine. On the horizon $z=100 \mathrm{~m}$ the cold intermediate layer (CIL), which covers a considerable part of 


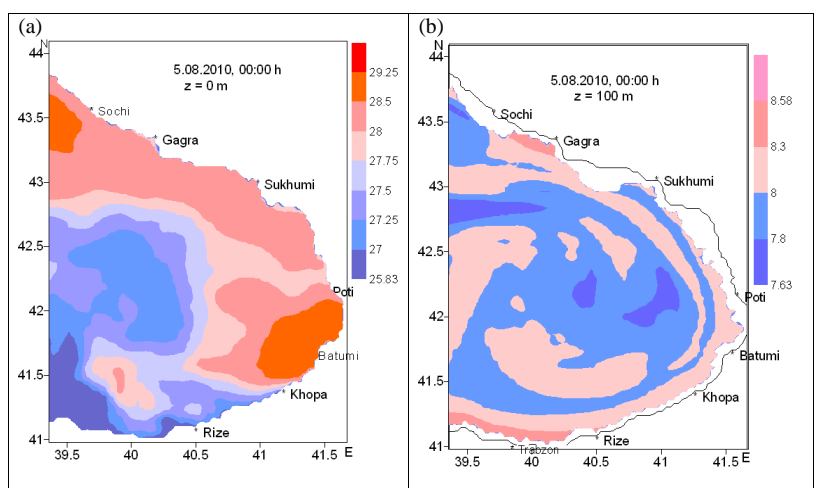

Fig. 3. Temperature field (deg. C) at 5 August 2010, 00:00 h on depths of $0 \mathrm{~m}$ (a) and $100 \mathrm{~m}$ (b).

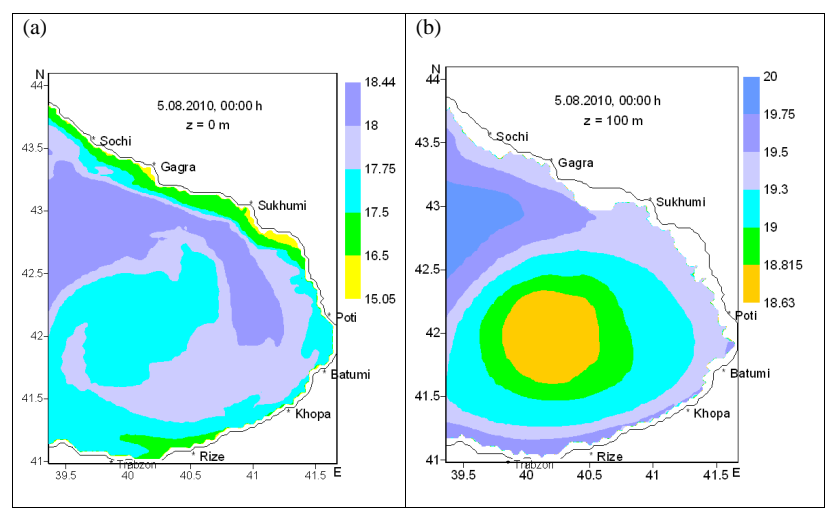

Fig. 4. Salinity field (psu) at 5 August 2010, 00:00 h on depths of $0 \mathrm{~m}$ (a) and $100 \mathrm{~m}$ (b).

the allocated water area is well observed (CIL is bounded with isotherm of $8^{\circ} \mathrm{C}$ ). The Batumi anticyclonic eddy makes appreciable impact on formation of salinity field in the considered area. General feature of this field is that the area of relatively low salinity waters coincides with allocation of anticyclonic eddy (compare Figs. 2 and 4). It is easy to explain this fact to that descending streams, which develop in the anticyclonic vortex, transfer fresher waters from the upper layer downwards (Stanev et al., 1988; Kordzadze, 1989).

The analysis of the wind stress fields for the forecasting period showed that atmospheric circulation significantly changed during this period.

The surface current fields after 24 and $72 \mathrm{~h}$ (time is counted from the initial moment of the forecast, $00: 00 \mathrm{~h}$, 6 August 2010), predicted by the RM-IG, are presented in Fig. 5 and the same fields predicted by the BSM of MHI at the same time moments are shown in Fig. 6.

The comparative analysis of Figs. 5 and 6 shows that forecasted regional circulation patterns obtained from RM-IG and BSM of MHI have common features, but it is evident that there are also distinctive features. The common feature is that accordance to both models the Batumi eddy is rather

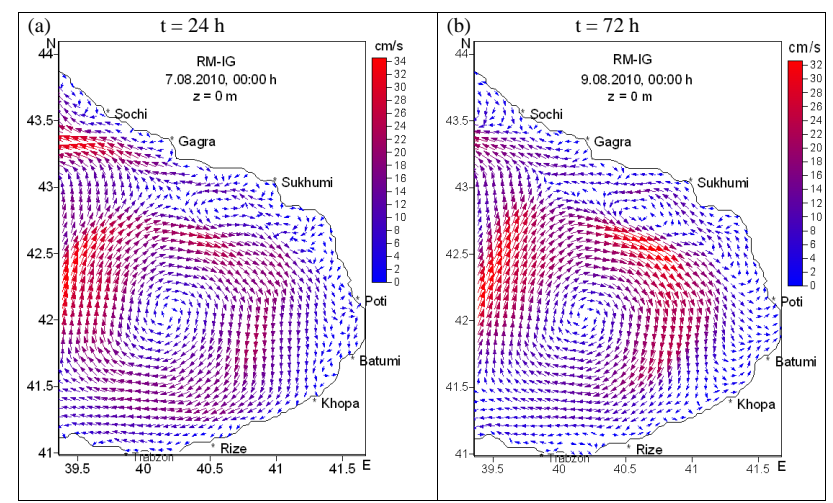

Fig. 5. The surface current fields predicted by RM-IG at $24 \mathrm{~h}$ (a) and $72 \mathrm{~h}$ (b). (The forecasting period is 6 August 2010, 00:00 h-9 August 2010, 00:00 h).

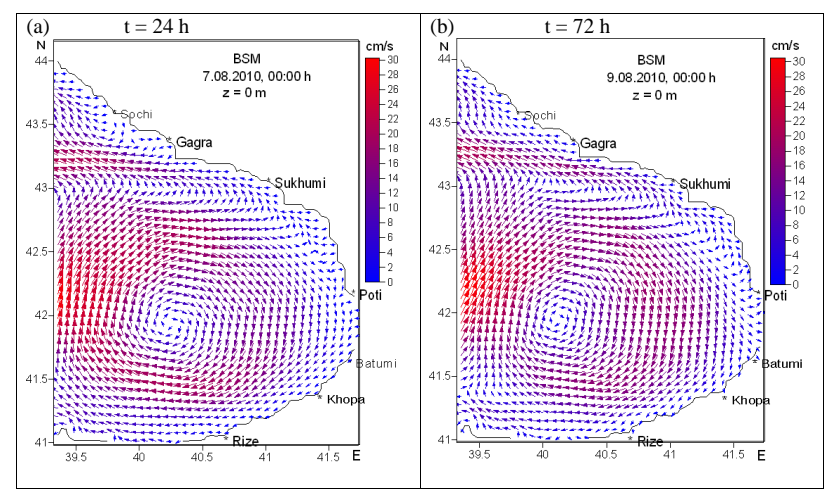

Fig. 6. The surface current fields predicted by BSM of MHI at $24 \mathrm{~h}$ (a) and $72 \mathrm{~h}$ (b) (the forecasting period is 6 August 2010, 00:00 h-9 August 2010, 00:00 h).

stable formation and it is observed during all forecasting period without strong changes despite nonstationarity of atmospheric forcing. It must be noted that formation of the anticyclonic eddy in the south-east part of the Black Sea basin and its stability in warm season is a well-known fact (Oguz et al, 1993; Korotaev et al, 2003). The main differences are in the narrow zone with width about $20-25 \mathrm{~km}$ along the Caucasian shoreline. The system of currents in this zone by results of RM-IG is characterized by the clear tendency to vortex formation of very small sizes and nonstationarity, whereas results of BSM specify about smoothness and practically non-stationary character of current in this zone. For example, unlike results of the BSM, in Fig. 5a formation of a small cyclonic eddy with a diameter about $15-20 \mathrm{~km}$ near the Sukhumi water area is observed at $t=24 \mathrm{~h}$, which becomes more clear to $t=72 \mathrm{~h}$. Likewise, near shoreline between Poti and Khopa the process of formation of very small unstable eddies is observed at $t=24 \mathrm{~h}$, but to the moment $t=72 \mathrm{~h}$ they are disappeared. The existence of rather unstable eddies of small sizes of cyclonic and anticyclonic characters near the 


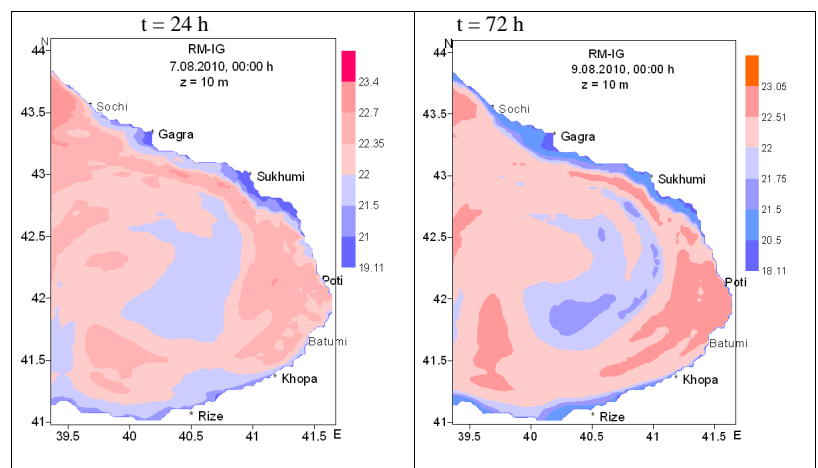

Fig. 7. The temperature fields (deg. C) on depth of $10 \mathrm{~m}$ predicted by RM-IG at $24 \mathrm{~h}$ and (b) $72 \mathrm{~h}$ (the forecasting period is 6 August 2010, 00:00 h-9 August 2010, 00:00 h).

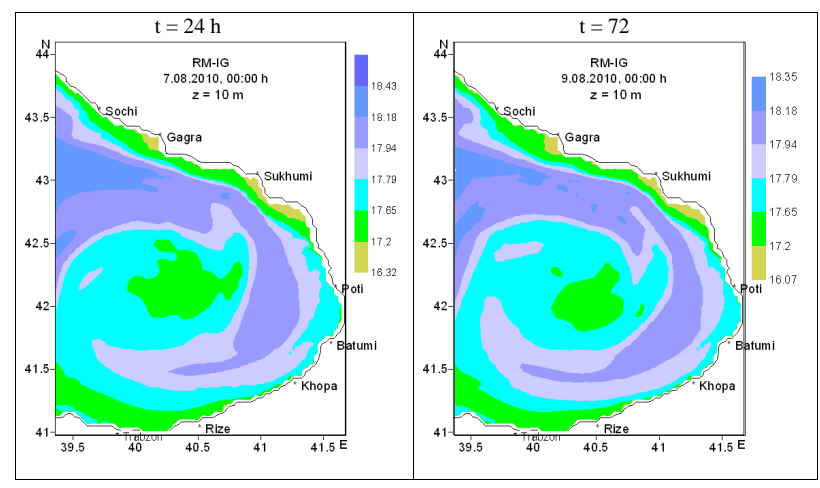

Fig. 8. The salinity fields (psu) on depth of $10 \mathrm{~m}$ predicted by RMIG at $24 \mathrm{~h}$ (a) and $72 \mathrm{~h} \mathrm{(b)} \mathrm{(the} \mathrm{forecasting} \mathrm{period} \mathrm{is} 6$ August 2010, 00:00 h-9 August 2010, 00:00 h).

Georgian shoreline is known from observations (Jaoshvili, 1986), but their identification by the mathematical models is possible by providing very high resolution, that is achieved in the RM-IG.

According to results of the RM-IG the narrow coastal zone of vortex formation makes certain impact on configuration of Batumi anticyclonic eddy and consequently on velocity distribution within the eddy. This zone plays a role of the interfering factor for the right peripheral current of the Batumi eddy to reach the Georgian coast. For example, from Fig. 5 is well visible, that the small anticyclonic eddy near Sukhumi water area represents an obstacle for peripheral current of the Batumi eddy and the current is passing through relatively narrow strip between the center of Batumi eddy and the small eddy near Sukhumi area. Therefore, here a zone of currents intensification with speeds about $30-32 \mathrm{~cm} \mathrm{~s}^{-1}$ is observed. The above phenomenon of regional circulation is not observed according to the results of BSM of MHI (Fig. 6).

Temperature and Salinity fields predicted by the RM-IG are presented in Figs. 7 and 8, and the same fields predicted by the BSM of MHI - in Figs. 9 and 10. In both cases these

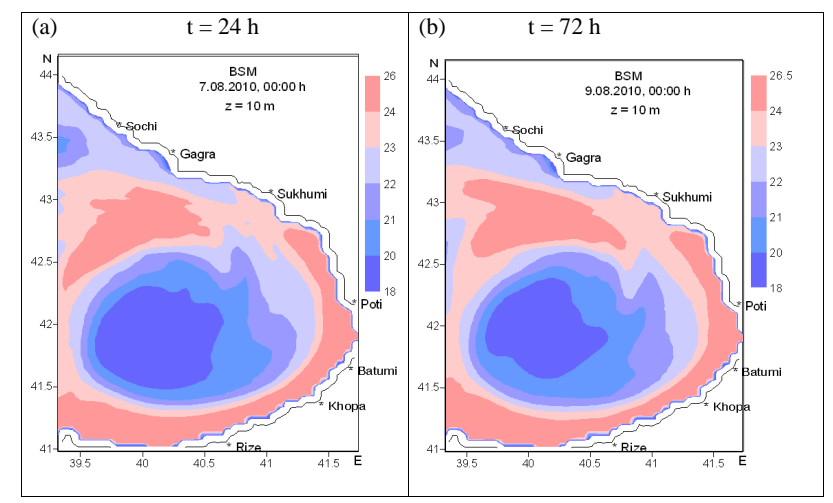

Fig. 9. The temperature fields (deg. C) on depth of $10 \mathrm{~m}$ predicted by BSM of MHI at $24 \mathrm{~h},(\mathbf{a})$ and $72 \mathrm{~h}$ (b). (the forecasting period is 6 August 2010, 00:00 h-9 August 2010, 00:00 h).

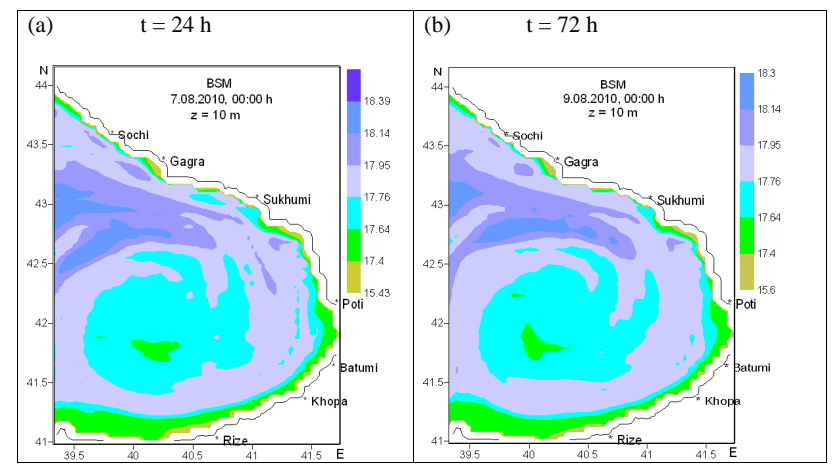

Fig. 10. The salinity fields (psu) on depth of $10 \mathrm{~m}$ predicted by BSM of $\mathrm{MHI}$ at $24 \mathrm{~h}$ (a) and $72 \mathrm{~h}$ (b) (the forecasting period is 6 August 2010, 00:00 h-9 August 2010, 00:00 h).

fields are shown on depth of $10 \mathrm{~m}$ and at moments 24 and $72 \mathrm{~h}$ after start of the forecast. Comparison between Figs. 7 and 9 shows some differences of temperature patterns computed on the basis RM-IG and BSM of MHI. From these Figures it is visible that in both cases colder waters are observed in the central area of the Batumi eddy than on its peripheries, but this feature is more expressed in the temperature field predicted by the BSM (Fig. 9). Comparison of Figs. 8 and 10 obviously demonstrate efficiency of the higher resolution model to simulate a salinity field in the coastal zone with higher adequacy. From Fig. 8 it is well visible the zone with high salinity, having a form of concave tongue, that apparently shows transfer of more salty waters from the open part of the sea into the Georgian water area by the Batumi eddy. The coarse resolution model is unable to reproduce this phenomenon (Fig. 10), whereas the fine intrusion of waters of different salinity is well resolved by the high resolution RMIG. 


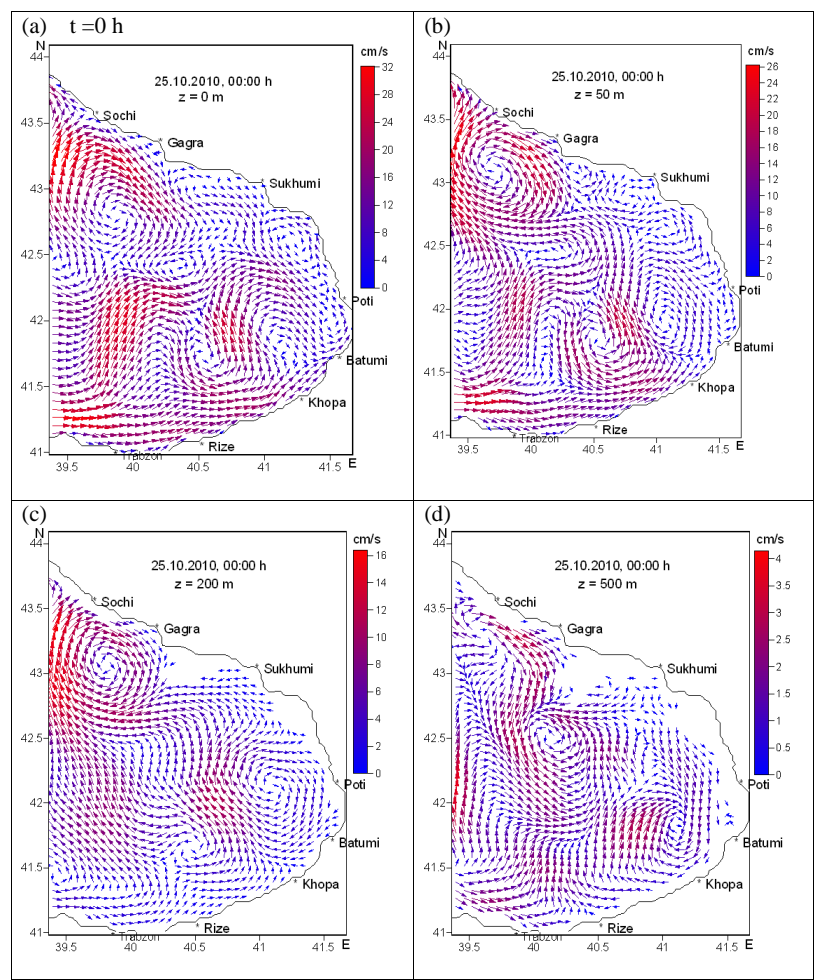

Fig. 11. Current field at 25 October 2010, 00:00 h on depths of $0 \mathrm{~m}$ (a), $50 \mathrm{~m} \mathrm{(b),} 200 \mathrm{~m}$ (c), and $500 \mathrm{~m}$ (d).

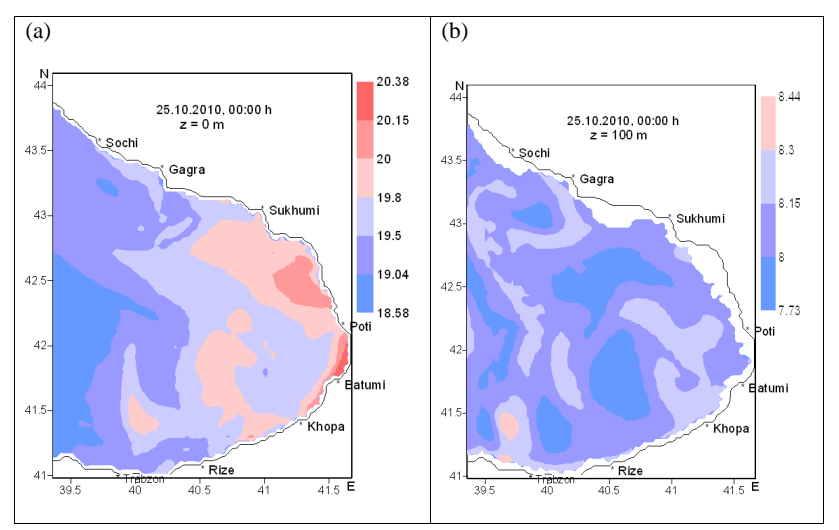

Fig. 12. Temperature field (deg. C) at 25 October 2010, 00:00 h on depths of $0 \mathrm{~m}$ (a) and $100 \mathrm{~m}$ (b).

\subsection{Forecast for autumn season}

The second example of the forecast concerns to the autumn, when the forecasting period was from $00: 00 \mathrm{~h}, 26$ October to 00:00 h, 29 October 2010. Like the previous case, integration of model equation system began one day earlier, at 00:00 h, 25 October.

The velocity pattern on some horizons at the beginning of integration is shown in Fig. 11. It is interesting to note

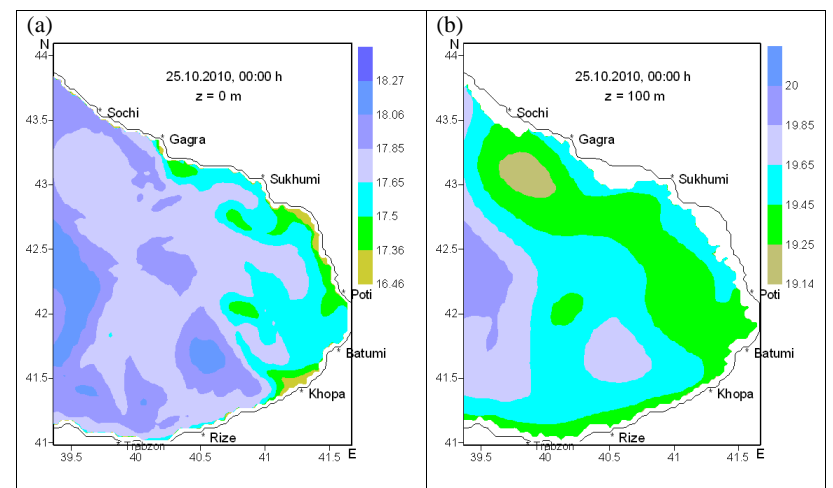

Fig. 13. Salinity field (psu) at 25 October $2010,00: 00 \mathrm{~h}$ on depths of $0 \mathrm{~m}$ (a) and $100 \mathrm{~m}$ (b).

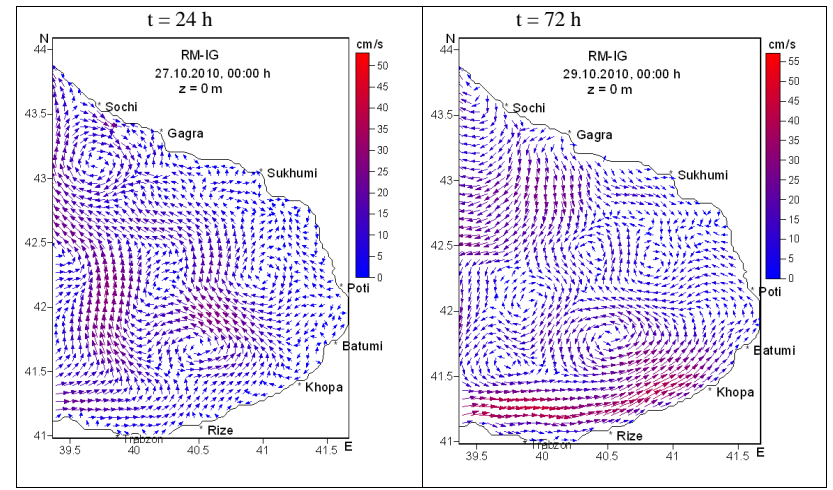

Fig. 14. The surface current fields predicted by RM-IG at $24 \mathrm{~h}$ (a) and $72 \mathrm{~h}$ (a) (the forecasting period is 26 October 2010, 00:00 h -29 October 2010, 00:00 h).

that the character of the regional circulation is quite different from circulation taking place on 5 August 2010 (see Fig. 2). The general structure of the regional circulation on $00: 00 \mathrm{~h}$, 25 October is characterized by formation of cyclonic and anticyclonic eddies of small sizes. Such structure is qualitatively kept up to deep levels with gradual reduction of the maximal speed of the flow from $32 \mathrm{~cm} \mathrm{~s}^{-1}$ up to $4 \mathrm{~cm} \mathrm{~s}^{-1}$ on horizon $z=500 \mathrm{~m}$. Accordingly, horizontal distributions of temperature and salinity fields (Figs. 12 and 13) differ from distribution of the same fields at 00:00, 5 August 2010 (see Figs. 3 and 4)

The Atmospheric wind above the regional area considerably changed during the forecasting interval.

The prognostic surface regional circulation patterns computed by the RM-IG are presented in Fig. 14 and the same patterns computed by the BSM of MHI are demonstrated in Fig. 15 at times of 24 and $72 \mathrm{~h}$ after start of the forecasting interval. Comparison of these two Figures shows that according to results of RM-IG, during the forecasting interval the regional circulation is characterized by intensive formation, deformation and disappearance of the small eddies with 


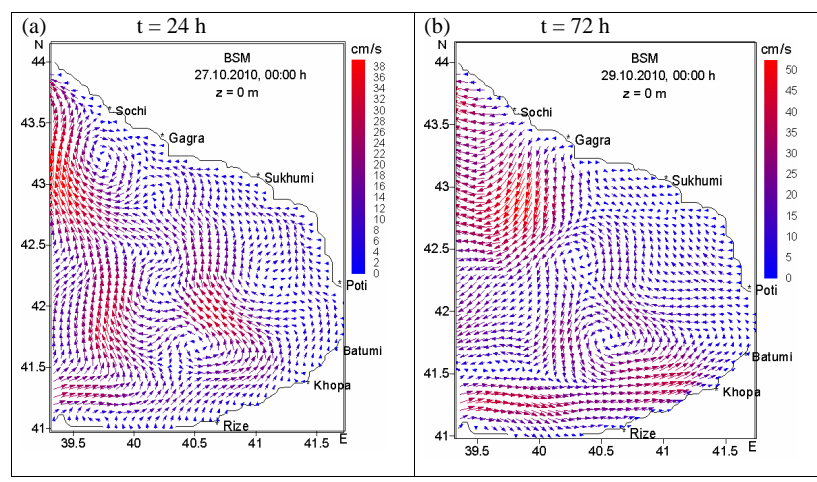

Fig. 15. The surface current fields predicted by BSM of MHI at $24 \mathrm{~h} \mathrm{(a)} \mathrm{and} 72 \mathrm{~h} \mathrm{(c)} \mathrm{(the} \mathrm{forecasting} \mathrm{period} \mathrm{is} 26$ October 2010, 00:00 h-29 October 2010, 00:00 h).

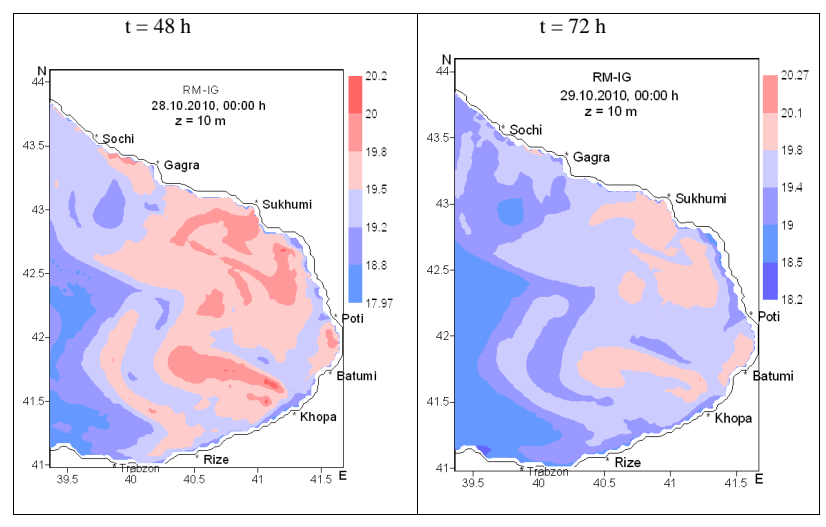

Fig. 16. The temperature fields (deg. C) on depth of $10 \mathrm{~m}$ predicted by RM-IG at $48 \mathrm{~h}$ (a) and $72 \mathrm{~h}$ (b) (forecasting period is 26 October 2010, 00:00 h-29 October 2010, 00:00 h).

diameter about $15-20 \mathrm{~km}$, while these properties of the regional circulation are much less expressed by results of the BSM of MHI. This fact once again directs us to the idea, that high resolution of numerical model is a major factor for improving identification of small unstable eddies which are permanently formed in the Georgian coastal zone.

The prognostic fields of temperature and salinity computed by the RM-IG are shown in Figs. 16 and 17 and the same fields predicted by the BSM are presented in Fig. 18 and 19. These fields are shown on the depth of $10 \mathrm{~m}$ after 48 and $72 \mathrm{~h}$ after the initial moment of the forecast. The comparative analysis of the fields predicted by both RM-IG and BSM shows, that in this case general features of temperature and salinity fields predicted by the both models are similar and qualitatively are close to each other, but they differ from each other quantitatively and with some separate nuances of the thermohaline fields.

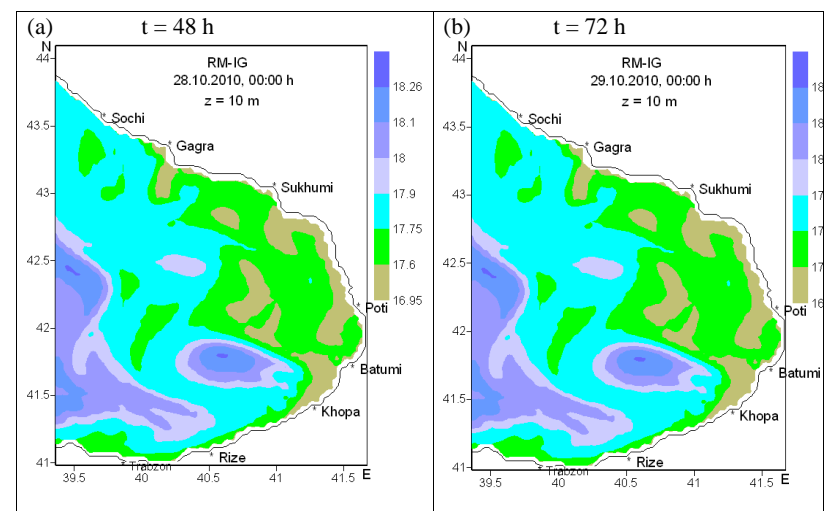

Fig. 17. The salinity fields (psu) on depth of $10 \mathrm{~m}$ predicted by RMIG at $48 \mathrm{~h} \mathrm{(a)} \mathrm{and} 72 \mathrm{~h}$ (b) (forecasting period is 26 October2010, 00:00 h-29 October2010, 00:00 h).

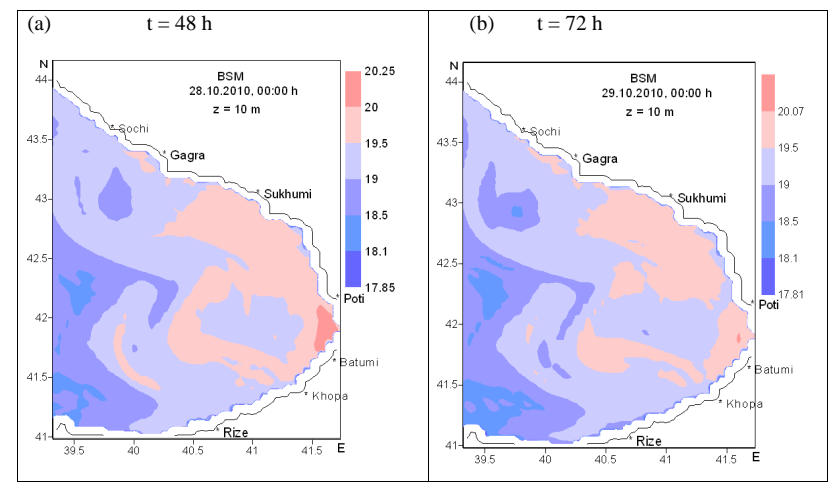

Fig. 18. The temperature fields (deg. C) on depth of $10 \mathrm{~m}$ predicted by BSM of MHI at $48 \mathrm{~h} \mathrm{(a)} \mathrm{and} 72 \mathrm{~h} \mathrm{(b)} \mathrm{(forecasting} \mathrm{period} \mathrm{is} 26$ October 2010, 00:00 h-29 October 2010, 00:00 h).

\section{Conclusions}

A regional forecasting system allowed to predict flow, temperature and salinity fields with $1 \mathrm{~km}$ spacing in the easternmost part of the Black Sea (including Georgian water area) is elaborated. The core of the forecasting system is the hydrostatic baroclinic regional model of the Black Sea dynamics developed at M. Nodia Institute of Geophysics, which is nested in the BSM of MHI.

The simulation and 3 days' forecasts of 3-D hydrophysical fields were performed for the summer and autumn seasons 2010 using the prognostic data of BSM of MHI and atmospheric dynamics model ALADIN.

The model simulation shows the seasonal differences between regional dynamical processes. Our results once again show already known fact that in summer the main element of the regional circulation in the considered area is the Batumi anticyclonic eddy. Unlike the summer circulation, in autumn the circulation has a vortical character with formation of cyclonic and anticyclonic eddies with diameters about 


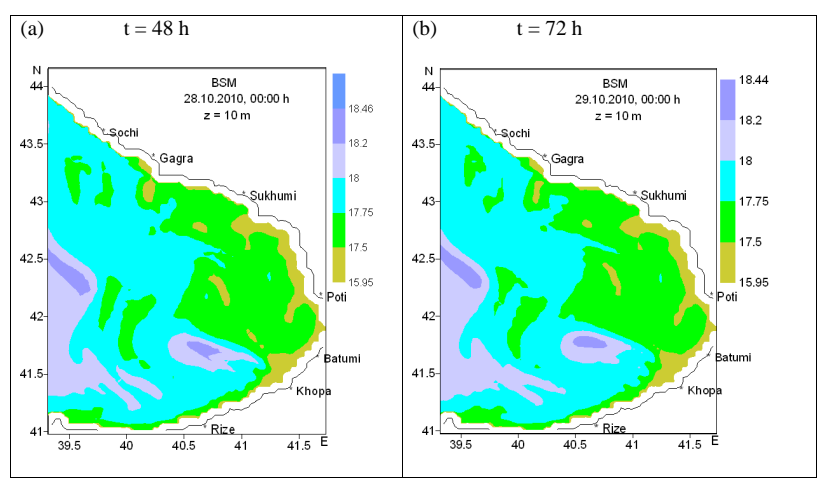

Fig. 19. The salinity fields (psu) on depth of $10 \mathrm{~m}$ predicted by $\mathrm{BSM}$ of MHI at $48 \mathrm{~h} \mathrm{(a)} \mathrm{and} 72 \mathrm{~h} \mathrm{(b)} \mathrm{(the} \mathrm{forecasting} \mathrm{period} \mathrm{is} 26$ October 2010, 00:00 h-29 October 2010, 00:00 h.).

$15-20 \mathrm{~km}$. These eddies are not stable formations and decompose or undergo some modifications in time.

The comparative analysis of predicted fields by both RMIG and BSM of MHI shows that to use the model with high resolution is very important factor for identification of coastal eddies of small sizes. In case of forecast of summer circulation more differences are observed in the narrow zone along Caucasus shoreline. In this near-shore zone the flow predicted by the RM-IG is characterized by nonstationarity and the tendency to form eddies of small sizes. Such character of the sea flow along the Georgian near-shore zone is known from observations (Jaoshvili, 1986). In case of forecast of autumn circulation considerable differences between the predicted current fields are observed: the vortex formation processes are more intensive at using of the RM-IG. It is also interesting fact that in summer, when the Batumi eddy is well developed, penetration of more salty waters from the open part of the Black Sea into the Georgian water area is distinctly observed by the results of the RM-IG.

The regional forecasting system presented in this study is a base for further development of a coupled modeling system allowing to predict not only dynamic processes, also different ecological processes including dispersion of oil and other polluting substances in the Georgian coastal zone in accidental situations. The hydrodynamic module of the coupled system - the RM-IG will be improved by taking into consideration a free surface and nonhydrostatic effects.

\section{Appendix A}

\section{Model equation system}

The RM-IG equation system, which describes the hydro and thermodynamic processes in the sea basin, is following:

$$
\frac{\partial u}{\partial t}+\operatorname{div} \mathbf{u} u-l v+\frac{1}{\rho_{0}} \frac{\partial p^{\prime}}{\partial x}=\nabla \mu \nabla u+\frac{\partial}{\partial z} \nu \frac{\partial u}{\partial z},
$$

$$
\begin{aligned}
& \frac{\partial v}{\partial t}+\operatorname{div} \mathbf{u} v+l u+\frac{1}{\rho_{0}} \frac{\partial p^{\prime}}{\partial y}=\nabla \mu \nabla v+\frac{\partial}{\partial z} v \frac{\partial v}{\partial z}, \\
& \frac{\partial p^{\prime}}{\partial z}=g \rho^{\prime}, \quad \operatorname{div} \mathbf{u}=0, \\
& \frac{\partial T^{\prime}}{\partial t}+\operatorname{div} \mathbf{u} T^{\prime}+\gamma_{T} \cdot w=\nabla \mu_{T} \nabla T^{\prime}+\frac{\partial}{\partial z} v_{T} \frac{\partial T^{\prime}}{\partial z}+\frac{\partial v_{T} \gamma_{T}}{\partial z} \\
& \quad-\frac{1}{c \rho_{0}} \frac{\partial I}{\partial z}-\frac{\partial \bar{T}}{\partial t},
\end{aligned}
$$$$
\frac{\partial S^{\prime}}{\partial t}+\operatorname{div} \mathbf{u} S^{\prime}+\gamma_{S} w=\nabla \mu_{S} \nabla S^{\prime}+\frac{\partial}{\partial z} v_{S} \frac{\partial S^{\prime}}{\partial z}+\frac{\partial \nu_{S} \gamma_{S}}{\partial z}-\frac{\partial \bar{S}}{\partial t},
$$$$
\rho^{\prime}=\alpha_{T} T^{\prime}+\alpha_{S} S^{\prime}, \quad \gamma_{T}=\frac{\partial \bar{T}}{\partial z}, \gamma_{S}=\frac{\partial \bar{S}}{\partial z},
$$$$
T=\bar{T}(z, t)+T^{\prime}, S=\bar{S}(z, t)+S^{\prime}, \rho=\bar{\rho}(z, t)+\rho^{\prime},
$$$$
p=\bar{p}(z, t)+p^{\prime},
$$$$
\nabla \mu \nabla=\frac{\partial}{\partial x} \mu \frac{\partial}{\partial x}+\frac{\partial}{\partial y} \mu \frac{\partial}{\partial y}, \quad I=\eta(1-A) I_{0} e^{-\alpha z},
$$

$I_{0}=a \sinh _{0}-b \sqrt{\sinh _{0}}, \sinh _{0}=\sin \varphi \sin \psi+\cos \varphi \cos \psi \cos \frac{\pi}{12} t$,

$\eta=1-(\tilde{a}+\tilde{b} \tilde{n}) \tilde{n}$.

$\alpha_{T}=\partial f / \partial \bar{T}=-10^{-3}(0.0035+0.00938 \bar{T}+0.0025 \bar{S})$,

$\alpha_{S}=\partial f / \partial \bar{S}=10^{-3} \cdot(0.802-0.002 \bar{T})$.

Here the following notations are used: $u, v$ and $w$ are the components of the current velocity vector along the axes $x$, $y$, and $z$, respectively (the axes $x, y$, and $z$ are directed eastward, northward, and vertically downward from the sea surface, respectively); $T^{\prime}, S^{\prime}, P^{\prime}, \rho^{\prime}$ are the deviations of temperature, salinity, pressure and density from their standard vertical distributions $\bar{T}, \bar{S}, \bar{P}, \bar{\rho} ; l=l_{0}+\beta . y$ is the Coriolis parameter, where $\beta=d l / d y ; g, c$, and $\rho_{0}$ are the gravitational acceleration, the specific heat capacity and average density of seawater, respectively; $\mu, \mu_{T, S}, \nu, \nu_{T, S}$ are the horizontal and vertical eddy viscosity, heat and salt diffusion coefficients, respectively; $I_{0}$ - the total flux of solar radiation at $z=0$; $A$ is the albedo of a sea surface, $h_{0}$ is the zenithal angle of the Sun; $\varphi$ is the geographical latitude, $\Psi$ is the parameter of declination of the Sun, $\eta$ is the factor which takes into account influence of a cloudiness on a total radiation and depends upon ball of nebulosity $\tilde{n}$ (Berlyand, 1960); $a, b, \tilde{a}, \tilde{b}$ are the empirical factors; $\alpha$ is the parameter of absorption of short-wave radiation by seawater. 


\section{Appendix B}

\section{Parameterization of the turbulent field}

Factors of horizontal viscosity and diffusion for temperature and salt $\mu, \mu_{T, S}$ where calculated by the formulas (Zilitinkevich and Monin, 1971)

$\mu=\Delta x \cdot \Delta y \sqrt{2\left(\frac{\partial u}{\partial x}\right)^{2}+\left(\frac{\partial u}{\partial y}+\frac{\partial v}{\partial x}\right)^{2}+2\left(\frac{\partial v}{\partial y}\right)^{2}}$,

$\mu_{T}=\frac{\mu}{c_{T}}, \mu_{S}=\frac{\mu_{S}}{c_{S}}$,

where $\Delta x$ and $\Delta y$ are horizontal grid steps along $x$ and $y$ respectively, $c_{T}$ and $c_{S}$ are some constants equal to 10 .

Factors of vertical turbulent diffusion for heat and salt $v_{T, S}$ were calculated by using the modified Oboukhov formula presented by Marchuk et al., (1980) as:

$\nu_{T, S}=(0.05 \mathrm{~h})^{2} \sqrt{\left(\frac{\partial u}{\partial z}\right)^{2}+\left(\frac{\partial v}{\partial z}\right)^{2}-\frac{g}{\rho_{0}} \frac{\partial \rho}{\partial z}}$.

Here $\mathrm{h}$ is the depth of the turbulent surface layer, which is defined by the first point $z_{m}$, in which following condition is satisfied:

$\left(0.05 z_{m}\right)^{2} \sqrt{\left(\frac{\partial u}{\partial z}\right)^{2}+\left(\frac{\partial v}{\partial z}\right)^{2} \frac{g}{\rho_{0}} \frac{\partial \rho}{\partial z}} \leq \nu_{T, S}^{0}, v_{T, S}^{0}=1 \mathrm{~cm}^{2} \mathrm{~s}^{-1}$

Vertical turbulent viscosity factor

$v= \begin{cases}50 \mathrm{~cm}^{2} \mathrm{~s}^{-1}, & z \leq 55 \mathrm{~m} \\ 10 \mathrm{~cm}^{2} \mathrm{~s}^{-1}, & z>55 \mathrm{~m}\end{cases}$

In case of unstable stratification, which might appear during integration of the equations $\left(\frac{\partial \rho}{\partial z}<0\right)$, the realization of this instability in the model is taken into account by increase of factor of turbulent diffusion $v_{T, S} 20$ times in appropriate columns from the surface to the bottom.

Acknowledgements. The Authors would like to thank the EU projects ARENA and ECOOP coordinators, G. Korotaev, Ole Krarup Leth, Paolo Oddo, A. Kubryakov, also all other colleagues, with which the authors fruitfully cooperated within the implementation of these projects.

Edited by: G. Korotaev

\section{References}

Berlyand, G. G.: Method of climatological calculation of the net radiation (in Russian), Meteorologia i gidrologia, Moscow, 6, 912, 1960.

Besiktepe, S. T.: Development of the regional forecasting system for the Black Sea, Proceed. of the "Second International Conference on Oceanography of Eastern Mediterranean and Black Sea: Similarities and Differences of Two Interconnected Basins", Ankara, Turkey, 14-18 October, 2002, 297-306, 2003.
Demetrashvili, D. I., Kvaratskhelia, D. U., and Gvelesiani, A. I.: On the vortical motions in the Black Sea obtained by the 3-D hydrothermodynamical numerical model, Adv. Geosci., 14, 295 299, doi:10.5194/adgeo-14-295-2008, 2008.

Demyshev, S. G. and Korotaev, G. K.: Numerical energy-balanced model of baroclinic currents in the ocean with bottom topography on the C-grid, in: Numerical Models and Results of Intercalibration Simulations in the Atlantic Ocean, edited by: Sarkisyan, A., Moscow, 163-231, 1992 (in Russian).

Jaoshvili, Sh. V.: The river alluvium and the beach formation of the Georgian Black Sea coast, Tbilisi, 155 pp., 1986 (in Russian).

Kordzadze, A. A.: Mathematical Problems of Solving Problems of Ocean Dynamics, VTs SO AN SSSR, Novosibirsk, 148 pp., 1982 (in Russian).

Kordzadze, A. A.: Mathematical modelling of sea currents (theory, algorithms, numerical experiments), Moscow, OVM AN SSSR, 218 pp., 1989 (in Russian).

Kordzadze, A. A. and Skiba, Yu. N.: Numerical calculations of the Black Sea with a three-dimensional model, Preprint, Computer Center, Siberian Branch of Russian Academy of Sciences, Novosibirsk, 32 pp., 1973 (in Russian).

Kordzadze, A. A. and Demetrashvili, D. I.: Numerical modeling of inner-annual variability of the hydrological regime of the Black Sea with taking into account of alternation of different types of the wind above its surface, Proceed. of Intern. Conference: "A year after Johanesburg-Ocean Governance and Sustainable Development: Ocean and Coasts - a Glimpse into the Future”. Kiev, Ukraine, October 27-30, 2003, 495-505, 2004.

Kordzadze, A. and Demetrashvili, D. I.: Simulation and forecast of hydrophysical fields in the part of the Georgian Black Sea coastal zone, J. Georgian Geoph. Soc., 12, 7-16, 2008.

Kordzadze, A. and Demetrashvili, D. I.: Modeling of dynamical processes in the Black Sea, Georgian Electronic Scientific Journal (GESJ): Physics, 1, 25-45, http://gesj.internet-academy.org. ge/phys/, 2010.

Kordzadze, A. A., Demetrashvili, D. I., and Surmava, A. A.: Numerical modeling of hydrophysical fields of the Black Sea under the conditions of alternation of atmospheric circulation processes, Izvestiya RAS, Atmospheric and Oceanic Physics, 44, 213-224, 2008.

Korotaev, G. K. and Eremeev, V. N.: Introduction in operative oceanography of the Black Sea, Sevastopol, HPC "EKOCIGidrofizika”, 382 pp., 2006 (in Russian).

Korotaev, G., Oguz, T., Nikiforov, A., and Koblinsky, C.: Seasonal, interannual, and mesoscale variability of the Black Sea upper layer circulation derived from altimeter data, J. Geophys. Res., 108, 3122, doi:10.1029/2002JC001508, 2003.

Korotaev, G., Cordoneanu, E., Dorofeev, V., Fomin, V., Grigoriev, A., Kordzadze, A., Kubriakov, A., Oguz, T., Ratner, Yu., Trukhchev, D., and Slabakov, H.: Near-operational Black Sea nowcasting/forecasting system, in: European Operational Oceanography: Present and Future, 4th EuroGOOS Conference, 6-9 June 2005, Brest, France, 269-275, 2006.

Kubryakov, A., Grigoriev, A., Kordzadze, A., Korotaev, G., Trukhchev, D., and Fomin, V.: Nowcasting/Forecasting subsystem of the circulation in the Black Sea nearshore regions, in: European Operational Oceanography: Present and Future, 4th EuroGOOS Conference, 6-9 June 2005, Brest, France, 605-610, 2006. 
Marchuk, G. I.: Numerical methods in weather prediction, Leningrad Gidrometeoizdat, 353 pp., 1967 (in Russian).

Marchuk, G. I.: Numerical Solution of Problems of Atmospheric and Oceanic Dynamics, Gidrometeoizdat, Leningrad, 303 pp., 1974 (in Russian).

Marchuk, G. I. and Kordzadze, A. A.: Perturbation theory and the formulation of inverse problems of ocean dynamics, Proceedengs of Tbilisi State University, Mathematica, Mekhanica, Astronomia, 259, 49-65, 1986 (in Russian).

Marchuk, G. I., Kordzadze, A. A., and Skiba, Yu. N.: Calculation of major hydrological fields of the Black Sea on the basis of the splitting method, Izvestiya USSR, Atmos. Ocean. Phys., 11, 379-393, 1975.

Marchuk, G. I., Kordzadze, A. A., and Zalesnyi, V. B.: Problem of mathematical modelling of sea and ocean currents, in: Differential and Integral Equations: Boundary-Value Problems, Tbilisi, 99-151, 1979 (in Russian).

Marchuk, G. I., Kochergin, V. P., Sarkisyan, A. S., Bubnov M. A., Zalesny, V. B., Klimok, V. I., Kordzadze, A. A., Kuznetsov, V. I., Protasov, A. B., Sukhorukov, B. A., Tsvetova, E. A., and Scherbakov, A. B.: Mathematical models of ocean circulation , Nauka, Novosibirsk, 288 pp., 1980 (in Russian).
Oguz T., Latun, V. S., Latif, M. A., Vladimirov, V. V., Sur, H. I., Markov, A. A., Ozsoy, E., Kotovshchikov, B. B., Eremeev, V. V., and Unluata, U.: Circulation in the surface and intermediate layers in the Black Sea, Deep. Sea Res., 40, 1597-1612, 1993.

Stanev, E., Truhchev, D., and Roussenov, V.: The Black Sea circulation and its numerical modelling, Sofia, Kliment Ohridski, 222 pp., 1988 (in Russian).

Sukhonosov, V. I.: On the correctness as a whole of a 3D problem of ocean dynamics, in: Mechanics of Inhomogeneous Continuous media, Computer Center, Siberian Branch of Russian Academy of Sciences, Novosibirsk, 52, 37-53, 1981 (in Russian).

Zilitinkevich, S. S. and Monin, A. S.: Turbulence in Dynamic Models of the Atmosphere, Nauka, Leningrad, 41 pp., 1971 (in Russian). 